RICE UNIVERSITY

\title{
Optimal Routing and Power Allocation for Wireless Networks with Imperfect Full-Duplex Nodes
}

\author{
by
}

\section{David A. Ramírez Domínguez}

A Thesis Submitted

IN PARTIAl Fulfillment of the

Requirements for the Degree

\section{Master of Science}

Approved, Thesis Committee:
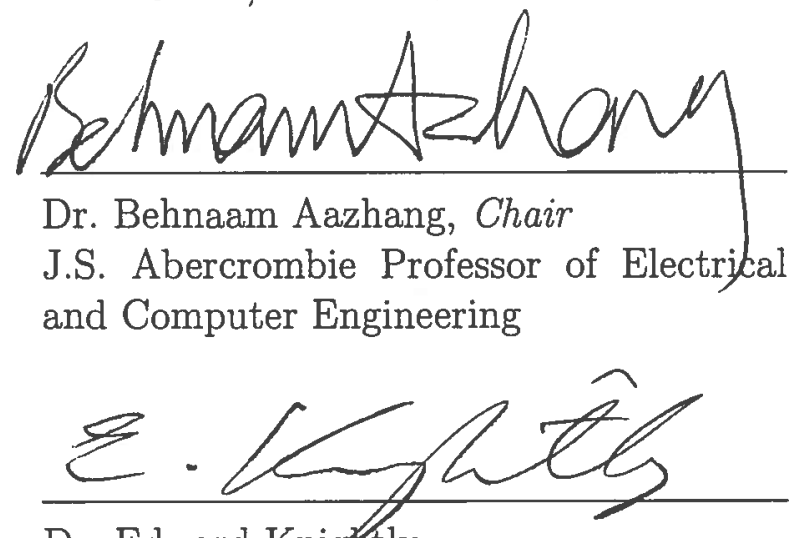

Dr. Edward Knigitity

Professor of Electrical and Computer Engineering

Dr. Ashutosh Sabharwal

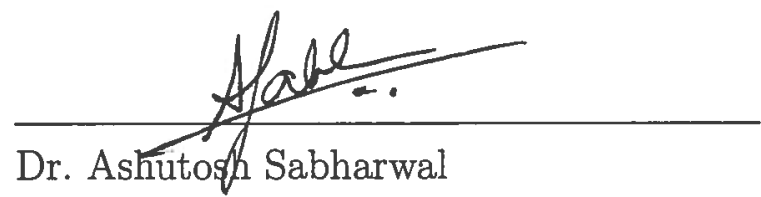

Professor of Electrical and Computer Engineering 


\begin{abstract}
Optimal Routing and Power Allocation for Wireless Networks with Imperfect Full-Duplex Nodes

by

David A. Ramírez Domínguez
\end{abstract}

We study a wireless full-duplex network with imperfect interference cancellation and solve the routing and power allocation problem in this network. We use a model that focuses on the effects of full-duplex by including residual self-interference and one hop interference while other interfering signals are considered negligible in comparison. We first solve the optimal power allocation for a fixed route. We then propose a priority-first search algorithm to find the joint route and power allocation to maximize throughput. The algorithm proposed has a non decomposable priority metric, but is efficiently evaluated by our solution for a fixed route. We analyze the performance of our solution in a more realistic model by deriving bounds between optimal solutions in both models. Through simulations we show that, even with imperfect interference cancellation, full-duplex achieves a higher throughput than half-duplex or direct transmission for moderate transmission power. 


\section{ACKNOWLEDGEMENTS}

Para mi familia, mis amigos, ma famille, mes amis, to the payday lunch group, and to my fellow group members for a never ending amount of support. To my teachers for sharing their knowledge.

"Factor means factor, solve means solve, and before you do anything else...look for the common factor" - M.H. 


\section{Contents}

Abstract $\quad$ ii

Acknowledgements $\quad$ iii

1 Introduction 1

2 Background 4

2.1 Network Model . . . . . . . . . . . . . . . . . . . . 4

2.2 Problem Statement . . . . . . . . . . . . . . . . . . . 7

2.3 Related Work . . . . . . . . . . . . . . . . 8

3 Power Allocation for a Fixed Route 11

3.1 Properties of Optimal Power Allocation . . . . . . . . . . . . . . . . . 12

3.2 Procedure for Optimal Power Allocation . . . . . . . . . . . . . . . . 14

4 Optimal Route and Power Allocation $\quad 19$

4.1 Priority-first Search Algorithms . . . . . . . . . . . . . . . . . 19

4.2 Algorithm for Routing and Power Allocation . . . . . . . . . . . . . 21

5 Performance in the Physical Model 27

5.1 Gap Between Optimal Solutions . . . . . . . . . . . . . . . . . 28

5.2 Performance of $\Delta$ with increasing $P_{\text {Max }} \ldots \ldots$. . . . . . . . . . 31

5.3 Performance of $\Delta$ with increasing $N$ in a Linear Network . . . . . . . 34

$6 \quad$ Numerical Results 39 
7 Conclusion 


\section{List of Figures}

2.1 Sample route $\mathbf{R}=\{S, y, z, D\}$ with 3 simultaneously transmitting nodes. Curved lines represent interfering signals and straight lines represent intended transmission. Normalized channel gains and achievable rates are shown above and below, respectively, the intended transmission. 5

2.2 Sample Network with $N=12$ with $S$ and $D$ labeled. Two sample routes are shown. The nodes of a sample route of cardinality 3 and $n=2$ is shown by connecting the nodes with a solid line. The nodes of a sample route of cardinality 4 and $n=3$ is shown by connecting the nodes with a dashed line. . . . . . . . . . . . . . 6

3.1 Sample plot of $P_{1}, P_{2}$, and $P_{3}$ as functions of $\omega$ with $\gamma_{1}=\gamma_{2}=\gamma_{3}=$ $0.02, h_{1,2}^{-1}=2, h_{2,3}^{-1}=4.5$, and $h_{3,4}^{-1}=6 \ldots \ldots \ldots$. . . . . . 16

5.1 Sample route $\mathbf{R}=\{S, y, z, D\}$ with 3 simultaneously transmitting nodes in the physical model. Curved lines represent interfering signals and straight lines represent intended transmission. The thick curved lines represent the interference considered in the physical model but not our model. Normalized channel gains and achievable rates are shown above and below, respectively, the intended transmission. . . .

6.1 Achieved throughput for optimal route and power allocation with $\alpha=$ 3. Throughput is represented on a logarithmic scale. . . . . . . . . . . 40

6.2 Average number of nodes in the optimal route as a function of $P_{M a x}$ for a fixed network size and for path-loss coefficient $\alpha=3$. . . . . . 
6.3 Solution from Fig. 6.1 in model including interferences from all transmitting nodes. Throughput is represented on a logarithmic scale. . .

6.4 Achieved throughput for optimal route and power allocation with $\alpha=$ 4. Throughput is represented on a logarithmic scale. . . . . . . . . . . 41

6.5 Achieved throughput for optimal route and power allocation with $\alpha=4$. 43

6.6 Performance of our solution in a model including interferences from all transmitting nodes with $\alpha=4 \ldots \ldots$. . . . . . . . . . 43 
Chapter 1

\section{Introduction}

Interference is a major obstacle to concurrent transmissions in a wireless network. Efficiently allocating network resources, such as power, can reduce interference and increase network performance. A substantial amount of research, as in [1-11], has been done in this regard, but the aspect of self-interference has been largely ignored. This can be attributed to self-interference being a characteristic of full-duplex networks, which have the ability to simultaneously transmit and receive over the same frequency. Full-duplex networks were thought to be feasible only in wired networks.

Through interference cancellation techniques authors in [12-15] have shown wireless full-duplex is feasible, but with less than twice the rate of half-duplex due to residual interference from imperfect self-interference cancellation. Feasibility results of imperfect full-duplex have motivated research in full-duplex networks with selfinterference.

We consider the problem of maximizing the throughput in an imperfect fullduplex wireless network, meaning that the self-interference cancellation that enables full-duplex is imperfect. In Chapter 2 we introduce a model that includes imperfect self-interference cancellation. Our model also considers one hop interference, but assumes other sources of interferences to be negligible when compared to self- 
interference and one hop interference. This simplification on the interfering sources gives us the tractability required for the procedure we propose in Chapter 3 to find an optimal solution in our model. In our work, we leverage our model to show that an optimal power allocation for a fixed route can be found by solving roots of polynomial equations. In Chapter 4 we propose a priority-first search type algorithm with a priority metric that cannot be decoupled. Our proposed algorithm solves the joint routing and power allocation problem to maximize throughput in a wireless network with full-duplex nodes that have imperfect interference cancellation.

In Chapter 5 we evaluate our model by taking the solution obtained by our algorithm and analyzing its performance in the physical model, as described in [22]. While our model only considers self-interference and one hop interference the physical model considers self-interference, one hop interference, and interference from any other active transmission. We show that the optimal solution from our algorithm is an upper bound for the optimal solution in the physical model. Asymptotic bounds on the difference between the optimal solution from our algorithm in our model and the optimal solution in the physical model are shown.

We analyze the behavior of throughput in our model when the maximum transmission power grows and for a growing network size. We show that in a fixed network at sufficiently large maximum transmit power becomes the optimal route is a direct transmission. To derive a bound on throughput for growing network size we use a linear network topology. We only use a linear network for the throughput analysis for a growing network size, all other results are independent of network topology. The second asymptotic bound we show is in a linear network with fixed maximum transmission power the throughput in our model is bounded above by a constant. Finally, through simulations we evaluate moderate scenarios and show the performance of our algorithm and evaluate the assumptions used in our model. 
Chapter 2

\section{Background}

In this Chapter we describe the general network model, define our problem, and finally review some of the relevant literature. Our network model includes imperfect self-interference, due to full-duplex operation of the wireless nodes, as well as one hop interference. Assuming such a model allows for tractability in solving the routing and power allocation problem, stated in the second section of this Chapter. Finally, in the third section we discuss research related to this thesis work.

\section{$2.1 \quad$ Network Model}

In this work we consider a network of $N$ wireless nodes operating in full-duplex, meaning that all nodes can simultaneously transmit and receive information on the same frequency. Distinguish the single source of information in the network as $S$, and the destination for that same information as $D$. It is assumed that the destination does not transmit information.

Define a route $\mathbf{R}$ as an ordered acyclic set, with cardinality $n+1$, of nodes in which the first element is $S$ and the final element is $D$. Define the set of all possible routes in a network of $N$ nodes as $\mathcal{R}$. Note that a route $\mathbf{R} \in \mathcal{R}$ is not required to include 


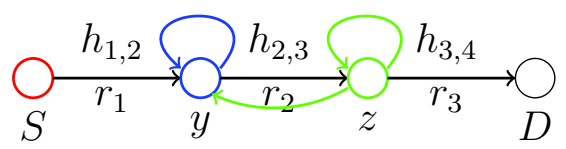

Figure 2.1: Sample route $\mathbf{R}=\{S, y, z, D\}$ with 3 simultaneously transmitting nodes. Curved lines represent interfering signals and straight lines represent intended transmission. Normalized channel gains and achievable rates are shown above and below, respectively, the intended transmission.

all $N$ nodes of the network. The order in the set $\mathbf{R}$ defines the transmission order, meaning that, for a route $\mathbf{R}$ the $i^{\text {th }}$ element in $\mathbf{R}$ transmits to the $(i+1)^{t h}$ element, while simultaneously the $(i-1)^{\text {th }}$ element transmits to the $i^{\text {th }}$ element. Nodes in a route $\mathbf{R}$ are said to be $k$ hops away from each other when they are $k$ elements apart from each other in R. In Fig. 2.1 node $z$ is two hops away from $S$ and only one hop away from $y$ and $D$.

In a route $\mathbf{R}$ of length $n+1$ the transmit power of node $i \in\{1, \ldots, n\}$ is defined as $P_{i}$. The power allocation for route $\mathbf{R}$ is defined to be $\mathbf{P}=\left\{P_{i}: i \in\{1, \ldots, n\}\right\}$. The set of feasible power allocations for a route $\mathbf{R}$ of length $n+1$ with $n$ transmitting nodes is defined as $\mathcal{P}=\left\{\mathbf{P}: 0 \leq P_{i} \leq P_{\text {max }} \forall P_{i} \in \mathbf{P}\right\}$. In Fig. 2.2 a sample wireless network with $N=12$ is shown.

Two different examples of routes with different value of $n$ are shown in Fig. 2.2. For any route it is assumed that $D$ does not transmit, hence we refer to the $n$ transmitting nodes of a route of cardinality $n+1$ as all the nodes that are not the destination $D$. The minimum $n$ of a route is $n=1, \mathbf{R}=\{S, D\}$, and we refer to such a route as a direct transmission.

When a node in the network is simultaneously transmitting and receiving information the node receives its own transmission creating self-interference. Results in [12] show a linear relation between a node's transmission power and self-interference. The use of self-interference cancellation techniques is the key component for enabling fullduplex operation. The self-interference cancellation techniques are imperfect; as such 


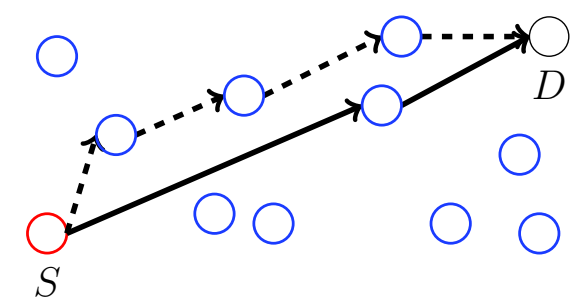

Figure 2.2: Sample Network with $N=12$ with $S$ and $D$ labeled. Two sample routes are shown. The nodes of a sample route of cardinality 3 and $n=2$ is shown by connecting the nodes with a solid line. The nodes of a sample route of cardinality 4 and $n=3$ is shown by connecting the nodes with a dashed line.

there exists residual self-interference. Interference cancellation techniques are improving, but current implementation results suggest the existence of a fundamental limit that impedes perfect self-interference cancellation.

For some node $i$ operating in full-duplex define the residual self-interference coefficient $\gamma_{i}$ to represent the efficiency of the self-interference cancellation technique used by node $i$. A perfect self-interference cancellation technique at node $i$ would achieve $\gamma_{i}=0$ and an imperfect technique achieves $0<\gamma_{i}<1$. Consider the results in [12] which reports a $20 \mathrm{~dB}$ difference between received transmission and the residual self-interference, the value of $20 \mathrm{~dB}$ would correspond to a coefficient of 0.01 .

To mitigate self-interference a node may be allocated a low transmit power. For a route $\mathbf{R}$ there are $n$ nodes simultaneously transmitting and can all be allocated different transmit powers. Consider route $\mathbf{R} \in \mathcal{R}$ and a power allocation $\mathbf{P} \in \mathcal{P}$ in which some node $i \in\{1, \ldots, n\}$ transmits at power $P_{i}$ and the node $i+1$ transmits at power $P_{i+1}$ with $P_{i} \ll P_{i+1}$ such that the interference originating at node $i+1$ affecting node $i$, referred to as one hop interference, can be comparable with the self-interference at $i$.

To maintain tractability in our model we will assume that interference originating at nodes that are further hops away is negligible compared to the combination of self-interference and one hop interference. Therefore, our model only considers self- 
interference and one hop interference. This is in contrast with the physical model that considers interference originating from all transmitting sources.

For a route $\mathbf{R} \in \mathcal{R}$ and power allocation $\mathbf{P} \in \mathcal{P}$ define the achievable transmission rate from element $i \in\{1, \ldots, n\}$ to element $i+1$ as

$$
r_{i}=\log \left(1+\frac{P_{i} h_{i, i+1}}{1+I_{i+1}}\right)
$$

where $h_{i, i+1}$ is the normalized channel gain between route elements $i$ and $i+1$. The normalized residual interference at node $i+1$ is defined as $I_{i+1}=P_{i+1} \gamma_{i+1}+$ $P_{i+2} h_{i+1, i+2}$, where the first term is the self-interference and the second term is the one hop interference. Normalization is done with respect to the noise power.

For a route $\mathbf{R} \in \mathcal{R}$ and a power allocation $\mathbf{P} \in \mathcal{P}$ we define throughput as $\rho(\mathbf{R}, \mathbf{P})=\min _{i \in\{1, \ldots, n\}} r_{i}$. That is to say, the throughput of a route operating with a power allocation is the minimum achieved rate among all nodes in the route.

\subsection{Problem Statement}

For a streaming application, which requires a continuous flow of information, it is beneficial to have a high throughput value. Therefore we present an algorithm that finds the optimal route $\mathbf{R}^{*} \in \mathcal{R}$ and associated optimal power allocation $\mathbf{P}^{*} \in \mathcal{P}$ such that $\rho\left(\mathbf{R}^{*}, \mathbf{P}^{*}\right) \geq \rho(\mathbf{R}, \mathbf{P}) \forall \mathbf{R} \in \mathcal{R}, \mathbf{P} \in \mathcal{P}$. The term optimal is with regards to throughput. Mathematically stated we seek to find a solution to

$$
\max \rho(\mathbf{R}, \mathbf{P}) \forall \mathbf{R} \in \mathcal{R}, \mathbf{P} \in \mathcal{P} \text {. }
$$

We take a systematic approach in solving this problem. In Section III we solve the problem of finding $\mathbf{P}^{*} \in \mathcal{P}$ given some route $\mathbf{R}$ such that $\rho\left(\mathbf{R}, \mathbf{P}^{*}\right) \geq \rho(\mathbf{R}, \mathbf{P}) \forall \mathbf{P} \in \mathcal{P}$. In Section IV we present an algorithm that finds both optimal route and power 
allocation, that is $\left(\mathbf{R}^{*}, \mathbf{P}^{*}\right)$, to maximize the throughput. In Section $\mathrm{V}$ we analyze the performance of the solution obtained by our algorithm when used in the physical model.

\subsection{Related Work}

The problem of routing and power allocation has been investigated in different scenarios. Often such work has assumed the use of half-duplex nodes, in which a wireless node can either transmit or receive but not do both simultaneously. Therefore the concept of scheduling arises. A schedule defines the time at which nodes should be transmitting and when should nodes be receiving. As such the joint routing, scheduling, and power allocation problem has been investigated in [1-5]. Scheduling can be done not only to fit the constraints of half-duplex operation but also to decrease interference. In [6] binary power allocation for a wireless network with interference is shown to be optimal. A binary power allocation is one in which a node will either transmit at maximum transmission power or transmit at 0 power, even when other transmission powers are possible. The result in [6] comes from the topology that is used in which there is a set of single receiver single transmitter pairs interfering with each other. When considering multiple sources of information the concept of fairness arises. Fairness is usually addressed with an optimization objective function that incurs a large penalty when a source can achieve a low utility when compared to other sources. Issues of resource allocation for fairness with interference are addressed in $[7-10]$.

Self-interference has been largely ignored by previous work. With full-duplex considered feasible only for wired networks, it is reasonable to assume only half-duplex operation in wireless networks and conduct research in models mostly without selfinterference. Following the results of [12-15] showing interference cancellation tech- 
niques that proved the feasibility of full-duplex there has been an increased interest in full-duplex models that consider self-interference.

The three node full-duplex network with self-interference has been thoroughly analyzed in [16-18]. Distributed routing algorithms in full-duplex networks with perfect self-interference cancellation are presented in [19]. In [20] implementation and simulation results show that imperfect full-duplex systems achieve better performance in networks larger than the three-node network. In [21] a random search style algorithm is presented to allocate resources in a wireless full-duplex network with selfinterference, but without considering interference cancellation. The work presented in this thesis focuses on routing and power allocation for an imperfect full-duplex network. The algorithm presented is not a random search, but rather a priority-first search algorithm inspired by Dijkstra's algorithm. Furthermore, we present bounds between our model and the often used physical model [22]. One of our results is restricted to a linear network. The linear network is used to restrict the topology of our network to enable analysis of the behavior of throughput for a growing $N$. Throughput in a linear network topology has been studied in [11,23-25], but not for aspects of routing and power allocation with imperfect full-duplex nodes.

Thus, our work differentiates from previous work by using a network model that accounts for imperfect self-interference arising from operating in full-duplex. Our wireless network thus moves past, with regards to number of nodes, the analysis of a three node network. Our algorithm considers self-interference, thus allocating power to negate the main deterrent of full-duplex while still benefiting of the increased capabilities. We then solve the jointly optimal routing and power allocation problem in our network model. Finally, we perform asymptotic analysis on throughput for increasing $P_{M a x}$ and increasing $N$. Our results show that routing becomes trivial at sufficiently large $P_{M a x}$ for any network and in a linear network throughput is bounded 
above by a function that tends to a constant at large $N$. 
Chapter 3

\section{Power Allocation for a Fixed Route}

In this Chapter we first introduce two lemmas that give us a necessary condition on the power allocation of a given route such that the achieved throughput is maximized. Using the two lemmas we introduce a procedure to find an optimal power allocation for a given route. We follow by presenting a theorem to prove the optimality of our procedure.

In the first lemma we show that when given a route and power allocation with unequal rates all rates can be equalized without lowering the throughput and with a

decrease in the sum transmit power of the route, defined as $\sum \mathbf{P}=\sum_{i=1}^{n} P_{i}$ which is the $L^{1}$ norm of the power allocation. Equalizing rates in the presence of interference is an efficient use of power and solves congestion, a problem usually addressed by higher communication layers.

Lemma 2 shows that even with self-interference and one hop interference the maximum throughput is achieved only if at least one node operates at maximum transmission power. Then we use the two lemmas to present a procedure to find $\mathbf{P}^{*} \in \mathcal{P}$ and prove optimality of the procedure. 


\subsection{Properties of Optimal Power Allocation}

We show in Lemma 1 that for a given power allocation with unequal rates has a throughput that may be increased. We also show that for a given power allocation with unequal rates the sum transmit power of the route can be decreased while maintaining the same throughput.

Lemma 1: Let $\mathbf{R}$ and $\mathbf{P} \in \mathcal{P}$ be a route and a corresponding power allocation. Choose $i \in\{1, \ldots, n\}$ so that $\rho(\mathbf{R}, \mathbf{P})=r_{i}$. If there exists $j \in\{1, \ldots, n\}$ with $r_{j}>r_{i}$, then there exists a $\mathbf{P}^{\prime} \in \mathcal{P}$ such that $\rho(\mathbf{R}, \mathbf{P}) \leq \rho\left(\mathbf{R}, \mathbf{P}^{\prime}\right)$ and $\sum \mathbf{P}^{\prime}<\sum \mathbf{P}$.

Proof. Let $i, j$ be as stated in Lemma 1, and first consider the case $i<j$. Then there exists a positive $\delta_{j} \in \mathbb{R}$ such that

$$
r_{i}<\log \left(1+\frac{\left(P_{j}-\delta_{j}\right) h_{j, j+1}}{1+I_{j+1}}\right)<r_{j}
$$

This reduces the self-interference at node $j$, therefore

$$
r_{i} \leq r_{j-1}<\log \left(1+\frac{P_{j-1} h_{j-1, j}}{1+\left(P_{j}-\delta_{j}\right) \gamma_{j}+P_{j+1} h_{j, j+1}}\right)
$$

where the term $I_{j}$ is expanded for clarity. Successively assign a positive $\delta_{k}$ for each $k \in\{i+1, \ldots, j-1\}$, such that $r_{i} \leq r_{k}$ with an allocated power of $P_{k}-\delta_{k}$. Therefore for any $k \in\{i+1, \ldots, j-1\}$

$$
r_{i}<\log \left(1+\frac{P_{k} h_{k, k+1}}{1+I_{k+1}-\delta_{k+1} \gamma_{k+1}-\delta_{k+2} h_{k+1, k+2}}\right) .
$$

Assign $\delta_{i}=0$ and $\delta_{l}=0 \forall l \in\{1, \ldots, n\}$ that were not previously assigned. Define $\mathbf{P}^{\prime}=\left\{P_{i}-\delta_{i}: i \in\{1, \ldots, n\}\right\}$. Since $0<\delta_{i} \forall i \in\{1, \ldots, n\}$, it is clear that $\sum \mathbf{P}^{\prime}<\sum \mathbf{P}$ and from (3.1), (3.2), and (3.3) it follows that $\rho(\mathbf{R}, \mathbf{P})<\rho\left(\mathbf{R}, \mathbf{P}^{\prime}\right)$.

When $j<i$ the same procedure can be followed such that $\sum \mathbf{P}^{\prime}<\sum \mathbf{P}$, but $I_{i}$ is 
the same for both $\mathbf{P}$ and $\mathbf{P}^{\prime}$, hence throughput does not increase nor decrease.

In perfect full-duplex, rate equalization does not necessarily lead to an increase in throughput even when in the first case of Lemma 1. Refer to Fig. 2.1 with $\gamma_{i}=$ $0 \forall i \in\{2,3\}$, thus perfect self-interference cancellation, and consider the case when $r_{1}=r_{2}<r_{3}$ in which reducing $P_{3}$ will not increase $r_{2}$, therefore the throughput does not increase.

Using Lemma 1 we introduce a necessary condition for optimality in Lemma 2 which guarantees that the maximum $\rho$ of a given $\mathbf{R}$ is achieved with at least one node transmitting at $P_{\text {Max }}$. After Lemma 2 we present our procedure to find $\mathbf{P}^{*} \in \mathcal{P}$.

Lemma 2: For a given $\mathbf{R}$ define $\mathbf{P}^{*} \in \mathcal{P}$ such that $\rho\left(\mathbf{R}, \mathbf{P}^{*}\right)=\max _{P \in \mathcal{P}}\left(\min _{i \in\{1, \ldots, n\}} r_{i}\right)$. There exists at least one $P_{i} \in \mathbf{P}^{*}$ such that $P_{i}=P_{\text {Max }}$.

Proof. To obtain a contradiction, assume that there exists $\mathbf{P}$ such that $\mathbf{P}=\left\{P_{i}\right.$ : $\left.P_{i}<P_{\text {Max }}, i \in\{1, \ldots, n\}\right\}$ so that $\rho\left(\mathbf{R}, \mathbf{P}^{*}\right)=\rho(\mathbf{R}, \mathbf{P})$. Use Lemma 1 to impose that $r_{i}=r_{j} \forall i, j \in\{1, \ldots, n\}$ without decreasing throughput. Rate equalization does not increase throughput since $\mathbf{R}^{*}$, from the statement, achieves the maximum throughput. Therefore since $P_{1}<P_{\max }$, there exists $\delta_{1} \in \mathbb{R}$ with $0<\delta_{1}<P_{\max }-P_{1}$ and

$$
r_{1}<\log \left(1+\frac{\left(P_{1}+\delta_{1}\right) h_{1,2}}{1+I_{2}}\right)=\eta_{1} .
$$

From properties of real numbers, continuity of the achievable rate function, and since $P_{2}<P_{\text {Max }}$ there exists $\delta_{2} \in \mathbb{R}$ with $0<\delta_{2} \leq P_{M a x}-P_{2}$ and

$$
r_{1}<\log \left(1+\frac{\left(P_{1}+\delta_{1}\right) h_{1,2}}{1+\left(P_{2}+\delta_{2}\right) \gamma_{2}+P_{3} h_{2,3}}\right)<\eta_{1}
$$

and

$$
r_{1}=r_{2}<\log \left(1+\frac{\left(P_{2}+\delta_{2}\right) h_{2,3}}{1+I_{3}}\right)
$$


Continue until values of $\delta_{i} \forall i \in\{1, \ldots, n\}$ are assigned and satisfy the inequality

$$
r_{1}<\log \left(1+\frac{\left(P_{i}+\delta_{i}\right) h_{i, i+1}}{1+I_{i+1}+\delta_{i+1} \gamma_{i+1}+\delta_{i+2} h_{i+1, i+2}}\right)
$$

Define $\mathbf{P}^{\prime}=\left\{P_{i}+\delta_{i}: i \in\{1, \ldots, n\}\right\}$. Then the contradiction $\rho\left(\mathbf{R}, \mathbf{P}^{*}\right)<\rho\left(\mathbf{R}, \mathbf{P}^{\prime}\right)$ follows.

Since our model focuses on interference from full-duplex transmissions, it is possible to not have a unique $\mathbf{P}^{*} \in \mathcal{P}$. Refer to Fig. 2.1 and suppose a $\mathbf{P}^{*} \in \mathcal{P}$ has been found for which $r_{1}=r_{2}=r_{3}$ and $P_{1}<P_{M a x}$, therefore $P_{1}$ can increase thus changing the power allocation without reducing throughput.

\subsection{Procedure for Optimal Power Allocation}

Lemma 1 shows that all rates can be equalized without lowering the achieved throughput while Lemma 2 shows that an optimal solution has at least one node transmitting at maximum power. To find a $\mathbf{P} \in \mathcal{P}$ that maximizes throughput it is not sufficient to force some $P_{i}=P_{M a x}$, since it does not solve for the values of $P_{j} \forall j \in\{1, \ldots, n\}$ nor does it guarantee maximizing $\rho$. Next, we show how using the two lemmas presented leads to our procedure for finding an optimal $\mathbf{P}^{*} \in \mathcal{P}$ for a given $\mathbf{R}$. Define the signal-to-interference-plus-noise ratio (SINR) at node $i \in\{2, \ldots, n+1\}$ as $\omega_{i}=P_{i} h_{i, i+1} /\left(1+I_{i+1}\right)$ and recall that $I_{n+1}=0$. As an example consider the route shown in Fig. 2.1 for which

$$
\omega_{4}=P_{3} / h_{3,4},
$$

and

$$
\omega_{3}=\frac{P_{2} h_{2,3}}{1+P_{3} \gamma_{3}}=\frac{P_{2} h_{2,3}}{1+\omega_{4} \gamma_{3} / h_{3,4}} .
$$


For the remaining node we can write the equation as

$$
\omega_{2}=\frac{P_{1} h_{1,2}}{1+\left(1+\omega_{4} \gamma_{3} / h_{3,4}\right) \omega_{3} \gamma_{2} / h_{2,3}+\omega_{4} h_{2,3} / h_{3,4}} .
$$

From Lemma 1 we know rate equalization is possible among all nodes without lowering the throughput, therefore $\omega=\omega_{i} \forall i \in\{1, \ldots, n\}$. Through algebraic manipulations rewrite these equations as

$$
\begin{gathered}
P_{3}=\frac{1}{h_{3,4}} \omega, \\
P_{2}=\frac{\gamma_{3}}{h_{2,3} h_{3,4}} \omega^{2}+\frac{1}{h_{2,3}} \omega,
\end{gathered}
$$

and

$$
P_{1}=\frac{1}{h_{1,2}}\left(\frac{\gamma_{3} \gamma_{2}}{h_{2,3} h_{3,4}} \omega^{3}+\left(\frac{h_{3,2}}{h_{3,4}}+\frac{\gamma_{2}}{h_{2,3}}\right) \omega^{2}+\omega\right) .
$$

From the previous three equations note that at a set power each equation is a polynomial of degree equal to the number of hops to reach the destination. The recursive equation that describes $P_{i}$ for a given route is

$$
P_{i}=\frac{\omega_{i}}{h_{i, i+1}}\left(1+\gamma_{i+1} P_{i+1}+h_{i+1, i+2} P_{i+2}\right)
$$

Remember that Lemma 2 indicates that at least one node will operate at $P_{\text {Max }}$. The following is the procedure we propose to find the optimal power allocation $\mathbf{P}^{*}$. First, using (3.14) recursively find the polynomial equation of $P_{i} \forall i \in\{1, \ldots, n\}$ as a function of $\omega$. Second, solve for the roots of the polynomial equations as functions of $\omega$ by setting $P_{i}=P_{\text {Max }} \forall i \in\{1, \ldots, n\}$. Third, find the maximum real root of the polynomial for element $i$ and label as $\omega^{i}$, which is the highest value possible of $\omega$ for that element. Fourth, find the smallest among these maximum real roots and label as $\omega^{*}=\min _{j}\left(\omega_{j}\right)$. Finally, to find the value of $P_{j} \forall j \in\{1, \ldots, n\}$ set $\omega_{j}=\omega^{*}$ in the 


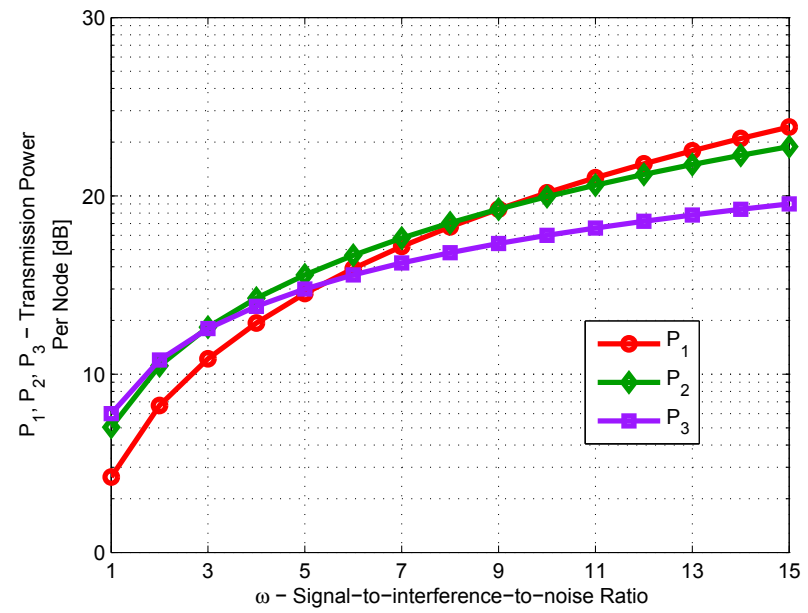

Figure 3.1: Sample plot of $P_{1}, P_{2}$, and $P_{3}$ as functions of $\omega$ with $\gamma_{1}=\gamma_{2}=\gamma_{3}=0.02$, $h_{1,2}^{-1}=2, h_{2,3}^{-1}=4.5$, and $h_{3,4}^{-1}=6$.

polynomial equation corresponding to $P_{j}$. Then $\mathbf{P}^{*}=\left\{P_{i}: i \in\{1, \ldots, n\}\right\}$, where $P_{i}$ is defined by (3.14) evaluated at $\omega^{*}$, achieves the maximum $\rho$ for the given route $\mathbf{R}$.

For illustration of the procedure refer to Fig. 3.1. In Fig. 3.1 we show the plot for $P_{1}, P_{2}$, and $P_{3}$ as functions of $\omega$. Arbitrary values of $\gamma_{1}=\gamma_{2}=\gamma_{3}=0.02$, $h_{1,2}^{-1}=2, h_{2,3}^{-1}=4.5$, and $h_{3,4}^{-1}=6$ were used for calculating this example. For a value of $P_{\text {Max }}<11$ the optimal power allocation would have $P_{1}=P_{\text {Max }}$. For a value of $11<P_{\text {Max }}<19$ the optimal power allocation would have $P_{2}=P_{\text {Max }}$. For $19<P_{\text {Max }}$ the optimal power allocation would have $P_{3}=P_{\text {Max }}$. When the roots of the polynomial equations are found, so are the regions in which a given node $i \in\{1, \ldots, n\}$ operates at $P_{i}=P_{M a x}$. When $\omega^{*}$ is found it will correspond to at least one $P_{i}=P_{M a x}$, and then the for all $i \neq j \in\{1, \ldots, n\}$ the values of $P_{j}$ can be allocated.

We have thus shown a procedure to find the optimal power allocation. The procedure we propose is enabled by our assumptions on interference. The following theorem shows optimality of the solution found by the procedure described above.

Theorem 1: For a given $\mathbf{R}$ and $\mathbf{P}^{*}$, as defined above, the throughput achieved is such that $\rho\left(\mathbf{R}, \mathbf{P}^{*}\right) \geq \rho\left(\mathbf{R}, \mathbf{P}^{\prime}\right) \forall \mathbf{P}^{\prime} \in \mathcal{P}$. 
Proof. Choose $j \in\{1, \ldots, n\}$ such that $P_{j}=P_{\text {Max }}$ and there is no $i<j \in\{1, . ., n\}$ such that $P_{i}=P_{\text {Max }}$. Existence is guaranteed from the construction of $\mathbf{P}^{*}$, but uniqueness is not. The only way to increase $r_{j}$ is to decrease $I_{j+1}$. Select an arbitrary $\delta_{j+1}>0$ such that the power allocation for node $j+1$ becomes $P_{j+1}^{\prime}=P_{j+1}^{*}-\delta_{j+1}>0$ then node $j+1$ achieves rate $r_{j+1}^{\prime}<r_{j+1}$ while increasing $r_{j}$. From construction $\rho\left(\mathbf{R}, \mathbf{P}^{*}\right)=r_{i} \forall i \in\{1, \ldots, n\}$, therefore $r_{j+1}^{\prime}<\rho\left(\mathbf{R}, \mathbf{P}^{*}\right)$. Then $I_{j+1}$ can be decreased to increase $r_{j+1}^{\prime}$ by the procedure above. Continue until the last transmitting node is reached, and for which the interference cannot be decreased. Define $\mathbf{P}^{\prime}=\left\{P_{i}^{*}-\delta_{i}\right.$ : $i \in\{1, \ldots, n\}\}$, then it follows that $\rho\left(\mathbf{R}, \mathbf{P}^{*}\right)>\rho\left(\mathbf{R}, \mathbf{P}^{\prime}\right) \forall \mathbf{P}^{\prime}$. Equality occurs when setting $\delta_{l}>0$ for any $l<j \in\{1, \ldots, n\}$ since it does not lower throughput.

Note that if $I_{n+1}$ were not a constant, in our model $I_{n+1}=0$, then the proof of Theorem 1 would not be valid. Therefore our procedure finds $\mathbf{P}^{*} \in \mathcal{P}$ for a given $\mathbf{R}$ in our model or in a model which assumes $I_{n+1}$ is constant.

Given a route $\mathbf{R}$ to solve by following our procedure an element $i \in\{1, \ldots, n\}$ has a polynomial equation of degree equal to $n-i+1$. The total number of polynomial equations to consider, and the largest degree of the polynomial equations, is equal to $n$. Therefore, the procedure has a complexity similar to a polynomial root finding algorithm.

We note that there exist formulas for the roots polynomials of degrees $\{1,2,3,4\}$. Therefore, when evaluating a route of $n \leq 4$ the formulas can be used directly and results compared to solve for an optimal power allocation. Empirical results in [26] and theoretical results in [27] reinforce the prevalence of routes of ideally 4 hops in different multiple hop scenarios. While there exist no formulas for roots of polynomials of degree 5 or higher, algorithms and techniques do exist in the literature that have been used to factor polynomials of degree 10,000, and higher, with random coefficients [28]. It is reasonable to assume that $N<10,000$ and therefore a polynomial of 
degree 10,000 would not be encountered. Furthermore, we are only interested in the maximum real root, a sub problem of the polynomial root finding problem. The development of a polynomial root finding technique falls outside the scope of this work, but we point out that many exist in the literature [29]. 
Chapter 4

\section{Optimal Route and Power Allocation}

To solve the optimal route and power allocation problem for a wireless network as the one described in Chapter 2 we propose a priority-first search algorithm. Our proposed algorithm is similar to Dijkstra's algorithm, with the main difference being the use of a non decomposable priority function. The use of a non decomposable priority function requires a new proof of optimality for the algorithm and results in distinct properties of the optimal solution.

\subsection{Priority-first Search Algorithms}

We propose a priority-first search algorithm that finds both the route and power allocation that maximize the throughput from $S$ to $D$. A popular example of a priority-first search is Dijkstra's algorithm [30]. The algorithm we propose differs from Dijkstra's algorithm by using a distinct priority metric, in particular one that is non decomposable. In this section we describe our algorithm and prove the optimality of the solution returned by our algorithm. We describe Dijkstra's algorithm in terms of a priority-first search to show the similarities and differences with respect to our proposed algorithm. 
We will first describe what is meant by "priority" and then we will describe how both algorithms work. To illustrate what is meant by priority, consider that in Dijkstra's algorithm the metric of priority is minimum total distance. Our algorithm modifies Dijkstra's algorithm by using maximum throughput as the metric of priority.

Dijsktra's algorithm does not require the distance to be the actual physical length between two nodes. Instead, distance can refer to the time it takes to travel between the two, a cost incurred by moving from one node to the other, or many other examples found throughout the literature [31,32].

We point out that maximum throughput problems are a subclass of minimum distance problems, as is known in the literature [33]. Therefore algorithms constructed specifically for maximum throughput are a subclass to the problem solved by Dijkstra's algorithm and can also be posed as a priority-first search. Hence we describe our algorithm and Dijkstra's algorithm as priority-first search and note that the same comparison can be carried out for other methods, e.g. Ford-Fulkerson algorithm, for maximum throughput algorithms [31].

Recall that the network has $N$ nodes. Assume any arbitrary indexing of all $N$ nodes. Note that a node $i \in\{1, \ldots, N\}$ should be understood as a node in the network, while a node $i \in\{1, . ., n\}$ should be understood as a node in a subset of nodes of the network.

Define a path $\pi_{i}$ as an ordered acyclic set of nodes in which the first element is the source $S$ and the final element is node $i \in\{1, \ldots, N\}$. By definition a path $\pi_{D}$ is also a route $\mathbf{R} \in \mathcal{R}$, but not all paths are routes.

For a path $\pi_{i}$ with $i \in\{1, \ldots, N\}$ the priority function in our algorithm is defined as $f\left(\pi_{i}\right)=\rho\left(\pi_{i}, \mathbf{P}^{*}\right)$, where $\mathbf{P}^{*}$ is found by the procedure defined in Chapter 3 . For any two nodes $j, k \in\{1, \ldots, N\}$ define the distance from $j$ to $k$ as $d_{j, k}$. The priority function in Dijkstra's algorithm for a path $\pi_{i}$ of cardinality $b+1$ is defined 
as $g\left(\pi_{i}\right)=\sum_{a \in\{1, \ldots, l\}} d_{a, a+1}$.

The proposed modification in the priority function is significant since in Dijkstra's algorithm the metric can be decomposed to the sum of the current value of the priority metric and the following decision. The decomposable property follows from the fact that the distance between any two nodes will not change as other nodes are added to the path. In our algorithm the metric is non decomposable since to calculate the metric, maximum throughput, all previous individual decisions that have been made are required. Therefore in our model the priority metric cannot be computed from the current value and the next decision. This follows from the allocated transmission power of one node affecting, at most, three different achievable rates.

\subsection{Algorithm for Routing and Power Allocation}

The algorithm initializes with the set of labeled nodes $\mathbf{L}=\{S\}$ and the set of unlabeled nodes $\mathbf{U}=\{j: j \neq S, j \in\{1, \ldots, N\}$. A node $i \in\{1, \ldots, N\}$ is said to be labeled when node $i$ is added to the set $\mathbf{L}$ and removed from the set $\mathbf{U}$.

The algorithm terminates whenever node $D$ has been labeled, that is when $D \in \mathbf{L}$. Throughout the execution of the algorithm a single path to node $i \in \mathbf{L}$ is kept in the set of paths $\Pi=\left\{\pi_{i}: i \in \mathbf{L}\right\}$. For a path $\pi_{i}$ and a node $v \in \mathbf{U}$ define the path extension $\pi_{i, v}=\left\{\pi_{i} \cup v\right\}$. Priority-first search algorithms can be described as a search for the path extension that achieves the highest priority among all possible path extensions.

We now define the iterative steps performed by the algorithm. The first step in the algorithm finds the path extension of highest priority $\pi_{i, u}^{*}=\max f\left(\pi_{j, v}\right) \forall \pi_{j} \in \Pi, v \in$ $\mathbf{U}$. The second step adds the path to node $u$ that was found, defined as $\pi_{u}=\pi_{i, u}^{*}$, to П. Then node $u$ is removed from $\mathbf{U}$, and node $u$ is added to $\mathbf{L}$. The algorithm then 
goes back to the first step and repeats, until $D \in \mathbf{L}$ and returns $\mathbf{R}^{*}=\pi_{D}$ and the associated $\mathbf{P}^{*}$. Dijkstra's algorithm follows the same iterative steps but utilizing the priority function $g\left(\pi_{i}\right)$ instead of $f\left(\pi_{i}\right)$, and is not required to compute or return any power allocation.

To prove that the algorithm terminates with an optimal solution in Lemma 3 we first prove that a path extension will not achieve a higher throughput than the path that it extends. In Lemma 4 we then show that at each iteration for any node $i \in\{1, \ldots, N\}$ the path $\pi_{i}$ found by the algorithm has a priority no less than the priority of any alternative path ending at node $i$. After both Lemmas a proof of optimality for the algorithm is given.

Lemma 3: Consider any path $\pi_{i}$ and an extension $\pi_{i, u}$ with $u \notin \pi_{i}$. The priorities of $\pi_{i}$ and $\pi_{i, u}$ are such that $f\left(\pi_{i}\right) \geq f\left(\pi_{i, u}\right)$.

Proof. To arrive at a contradiction assume that $f\left(\pi_{i}\right)<f\left(\pi_{i, u}\right)$. Define $j+1$ as the cardinality of $\pi_{i}$ and $l+1$ as the cardinality of $\pi_{i, u}$. Recall that from Lemma 1 all rates in a path with optimal power allocation are equal, therefore

$$
\log \left(1+P_{j} h_{j, j+1}\right)<\log \left(1+P_{l} h_{l, l+1}\right)
$$

If this is true, then from Lemma 1 it should also be true that

$$
\log \left(1+P_{j} h_{j, j+1}\right)<\log \left(1+\frac{P_{l-1} h_{l-1, l}}{1+I_{l}}\right) .
$$

Note that index $l-1$ represents the same node as index $j$ therefore $h_{j, j+1}=h_{l-1, l}$. Since $I_{l}>0$ then $P_{l-1}>P_{j}$ must be true for (4.2) to be true. Again, from Lemma 1 we can consider the achievable rates $r_{j-1}$ and $r_{l-2}$ which must also satisfy

$$
\log \left(1+\frac{P_{j-1} h_{j-1, j}}{1+I_{j}}\right)<\log \left(1+\frac{P_{l-2} h_{l-2, l-1}}{1+I_{l-1}}\right)
$$


such that the assumption $f\left(\pi_{i}\right)<f\left(\pi_{i, u}\right)$ is true. Since $h_{j-1, j}=h_{l-2, l-1}$ and $P_{l-1}>P_{j}$ then it follows that $I_{j}<I_{l-1}$ therefore $P_{j-1}<P_{l-2}$. Continue recursively comparing rates that correspond to the same nodes. Lemma 2 guarantees that there is at least one node operating at $P_{M a x}$, define $k \in\{1, \ldots, j\}$ as the element for which $P_{k}=P_{M a x}$. Define $k^{\prime} \in\{1, \ldots, l\}$ as the index that corresponds to the same node as the index $k \in\{1, \ldots, j\}$, then

$$
\log \left(1+\frac{P_{k} h_{k, k+1}}{1+I_{k+1}}\right)<\log \left(1+\frac{P_{k^{\prime}} h_{k^{\prime}, k^{\prime}+1}}{1+I_{k^{\prime}+1}}\right) .
$$

As before note that $h_{k, k+1}=h_{k^{\prime}, k^{\prime}+1}$. Therefore from the recursive argument $I_{k+1}<$ $I_{k^{\prime}+1}$ the contradiction $P_{M a x}=P_{k}<P_{k^{\prime}}$ follows. Therefore, any extension done to path $\pi_{i}$ will not achieve a higher priority.

Lemma 3 shows that the throughput of a path will not increase with a path extension. We have thus shown, in Lemma 3, that the priority function in our algorithm is monotonic, which is the case also for Dijkstra's algorithm. While Dijkstra's algorithm uses a monotonic increasing function, the function in our algorithm is monotonic decreasing.

We use the result from Lemma 3 to show in Lemma 4 how the algorithm at any step will have the path of highest priority to any node in $\mathbf{L}$. Afterwards Lemma 4 is used to show the optimality of the algorithm.

Lemma 4: Consider the path $\pi_{i}$ that has been added to $\Pi$ for some iteration of the algorithm. Any alternative path $\pi_{i}^{\prime}$ has priority such that $f\left(\pi_{i}\right) \geq f\left(\pi_{i}^{\prime}\right)$.

Proof. To prove by induction note that at initialization the claim in Lemma 4 is true since $\pi_{S}$ is the only path in $\Pi$, and by definition a path cannot end in $S$. After the first iteration define $\pi_{j}$ as the path added to $\Pi$. To show that $\pi_{j}$ is the path of highest priority to node $j \in\{1, \ldots, N\}$ note that any other path $\pi_{k}^{\prime}=\{S, k\}: k \in \mathbf{U}$ achieves 
a priority $f\left(\pi_{j}\right) \geq f\left(\pi_{k}^{\prime}\right)$, since if $f\left(\pi_{j}\right)<f\left(\pi_{k}^{\prime}\right)$ then $\pi_{k}^{\prime}$ would have been added to $\Pi$ in the first iteration. From Lemma 3 it follows that any extension of $\pi_{k}^{\prime}$ will not increase the priority, hence any extensions that lead to node $j$ will not have a priority higher than $\pi_{j}$.

After $m$ iterations define $\pi_{i}$ as the path added to $\Pi$ and $\tilde{\pi}_{i}$ as the path that was extended to get to node $i \in\{1, \ldots, N\}$, therefore $\pi_{i}=\left\{\tilde{\pi}_{i}, i\right\}$. A path to node $i$ can be constructed in the following ways: first a path to $i$ can be found from $\tilde{\pi}_{i}$ through a different extension than the one found by the algorithm, second a different path in $\Pi$ that is not $\tilde{\pi}_{i}$ could be extended to node $i$, third a path through paths not in $\Pi$ can be selected to arrive at node $i$. We go through all three possibilities to show that $\pi_{i}$ achieves a higher priority than any alternative path to node $i$.

From the algorithm any extension to $\tilde{\pi}_{i}$ that does not result in $\pi_{i}$ does not have a priority higher than $\pi_{i}$, hence from Lemma 3 extending the alternative will not achieve a higher priority. Consider any path $\pi_{k}^{\prime} \neq \tilde{\pi}_{i} \in \Pi$, from the algorithm at iteration $m$ there is no extension to $\pi_{k}^{\prime}$ that achieves a priority higher than $\pi_{i}$, else $\pi_{i}$ would not have been selected by the algorithm. From Lemma 3 it follows that any set of extensions to $\pi_{k}^{\prime}$ that end at node $i$ will not achieve a higher throughput than $\pi_{i}$. From the algorithm any path that goes through elements of $\mathbf{U}$ does not achieve a higher priority than any path in $\Pi$, else they would be in $\Pi$.

Having proved the statement is true at initialization, for the first iteration, and for iteration $m$ it then follows that the statement holds for any iteration of the algorithm.

Lemma 4 shows that at any iteration of the algorithm the path $\pi_{i} \in \Pi$ is the path of highest priority to node $i \in \mathbf{L}$ among any other possible path to node $i$. From Lemma 4 it follows that any path $\pi_{i} \in \Pi$ is the throughput optimal path from $S$ to $i \in \mathbf{L}$. 
Simply stated, the algorithm will always choose the path of highest priority. For Dijkstra's algorithm, that means the path of minimum distance, while in our algorithm it means selecting the path of highest throughput. The result of Lemma 4 is used to show the optimality of the algorithm in Theorem 2.

Theorem 2: For any network in which a route exists the algorithm terminates, and does so with the throughput optimal route and power allocation.

Proof. If $\mathbf{U}$ is non empty and $D \notin \mathbf{L}$ then the algorithm continues with the extraction of a single element of $\mathbf{U}$ at each iteration. Since at initialization $D \in \mathbf{U}$ and $|\mathbf{U}|=$ $N-1$ then the terminating condition is eventually met. The algorithm terminates when $D \in \mathbf{L}$, at this iteration a path $\pi_{D}$ has been added to $\Pi$. Since $\pi_{D}$ ends at $D$ it is also a route and from Lemma 4 there is no alternative path with a higher priority.

A proof of Dijkstra's algorithm as a priority-first search algorithm follows by using the appropriate priority function and with Lemma 3 and Lemma 4 proved in a manner appropriate for the priority function.

At each iteration the algorithm moves one element from $\mathbf{U}$ to $\mathbf{L}$, therefore the algorithm terminates in at most $N-1$ iterations. At iteration $i \in\{1, \ldots, N-1\}$ a total of $N-i$ possible paths need to be evaluated, each with at most $i+1$ elements. Recall that the length of the path dictates the highest degree of the polynomials that must be solved to find the optimal power allocation. With regards to complexity of polynomial root finding the worst case is a polynomial of degree $N$. Note that if a polynomial of degree $N$ is being considered then it is obviously the optimal route, else the algorithm would not arrive at a path of that length, additionally there is only a single path of cardinality $N$ to potentially be evaluated by the algorithm. In the worst case scenario a total of $\sum_{i=1}^{N}(N-i)$ evaluations of the priority function are required. 
An implementation of this algorithm requires network information, such as channel gains and residual self-interference coefficients, similar to that required by an implementation of Dijkstra's algorithm, of which many exist in the literature. While the amount of network information required is similar due to the priority function being non decomposable to compute the throughput of a path extension it is not sufficient to learn only the throughput of a current path and the next channel gain. Hence, the distribution of such network information is a higher burden if our algorithm were to be implemented.

Due to the interference model our priority function does not have certain properties that the priority function used in Dijkstra's algorithm does. Refer to Fig. 2.1 and assume that at termination of the algorithm the route $\pi_{D}=\{S, y, z, D\}$ is found as the optimal route. For Dijkstra's algorithm a subset of $\pi_{D}$ that maintains the same order as $\pi_{D}$, such as the set $\{y, z, D\}$ will also be the optimal route from node $y$ to the destination $D$. Additionally the inverse subset $\{D, z, y\}$ will be the optimal set that goes from the destination $D$ to node $y$. For our model, due to interference, such a subset of the optimal route or an inverse subset is not necessarily optimal. Since the coefficients and degrees of the set of polynomials used to find the optimal power allocation change and therefore the value of $\omega^{*}$ may change as well.

Thus we have presented a procedure to find the optimal power allocation when a route is given. We built on the procedure for optimal power allocation to construct a priority-first search algorithm. For the algorithm we show the priority function is monotonic and therefore the result of the algorithm is optimal. 
Chapter 5

\section{Performance in the Physical Model}

We have shown a procedure that finds an optimal power allocation for our model of a wireless network with imperfect full-duplex nodes. Our model simplified the effects of interference by limiting it to self-interference and one hop interference, both of which arise from imperfect full-duplex. The simplification proved to be fundamental in enabling our proposed procedure to find an optimal solution. We built on this procedure and presented a priority-first search algorithm to find the jointly optimal route and power allocation to maximize the throughput.

When the interference assumptions from our model are extended to include more interferers then our procedure may no longer be feasible. In particular, our procedure still finds an optimal solution if reception at node $i \in\{2, \ldots, n+1\}$ is interfered also by

any node $j \in\{i+2, \ldots, n\}$. When considering interference from any $k \in\{1, . ., i-2\}$ then an equation for $P_{i-1}$ depending only on $\omega$ cannot be written and therefore our proposed procedure cannot be applied. 


\subsection{Gap Between Optimal Solutions}

In this section we study how the solution from our procedure performs in the physical model as introduced in [22]. In the physical model for a route $\mathbf{R}$ and power allocation $\mathbf{P}$ and $i \in\{1, \ldots, n\}$ the achievable rate $r_{i}$ is affected by all other transmitting nodes. Our model gains tractability by limiting the sources of interference that are considered. Thus, analyzing the performance of the solution from our algorithm evaluated in the physical model allows for an analysis of what is paid to gain tractability.

Recall that in our model the achievable rate $r_{i}$ is only affected by interference originating from the $(i+1)^{t h}$ and $(i+2)^{t h}$ elements in $\mathbf{R}$. For a receiving node $k \in\{1, \ldots, n\}$ define the interferers considered in the physical model but not our model as the complement set of interferers $\mathbf{I}_{k}=\{j: j \neq\{k-1, k, k+1\} \forall j \in\{1, \ldots, n\}\}$.

The physical model also considers a transmission to be successful if the SINR at a receiving node is greater than a fixed SINR threshold. To maintain the assumptions from the initial model the SINR threshold is set to 0. Note that the proof of optimality of our algorithm requires the existence of a route. Including an SINR threshold other than 0 would reduce the connectivity in the network, which may translate into a shorter execution time but may result in a network with no feasible route.

In the physical model for a route $\mathbf{R}$ and power allocation $\mathbf{P}$ the achievable transmission rate of element $i \in\{1, \ldots, n\}$ to element $i+1$ is defined as

$$
\hat{r}_{i}=\log \left(1+\frac{P_{i} h_{i, i+1}}{1+I_{i+1}+\sum_{\left\{j \in \mathbf{I}_{i+1}\right\}} P_{j} h_{j, i+1}}\right) .
$$

Note that $r_{i} \geq \hat{r}_{i} \forall i \in\{1, \ldots, n\}$, with equality when $\mathbf{I}_{i+1}$ is empty or $P_{j}=0 \forall j \in \mathbf{I}_{i+1}$.

Define throughput in the physical model as $\hat{\rho}(\mathbf{R}, \mathbf{P})=\min _{i \in\{1, \ldots, n\}} \hat{r}_{i}$. Define the optimal throughput in the physical model as $\hat{\rho}\left(\hat{\mathbf{R}}^{*}, \hat{\mathbf{P}}^{*}\right) \geq \hat{\rho}(\mathbf{R}, \mathbf{P}) \forall \mathbf{R} \in \mathcal{R}, \mathbf{P} \in \mathcal{P}$. Note that $\hat{\mathbf{R}}^{*}$ and $\hat{\mathbf{P}}^{*}$ are not necessarily equal to $\mathbf{R}^{*}$ and $\mathbf{P}^{*}$, the optimal route and 


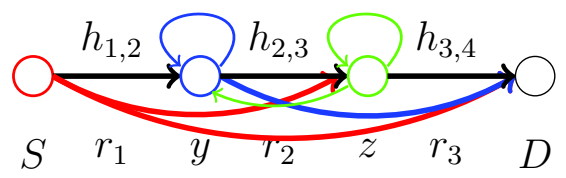

Figure 5.1: Sample route $\mathbf{R}=\{S, y, z, D\}$ with 3 simultaneously transmitting nodes in the physical model. Curved lines represent interfering signals and straight lines represent intended transmission. The thick curved lines represent the interference considered in the physical model but not our model. Normalized channel gains and achievable rates are shown above and below, respectively, the intended transmission.

power allocation in our model.

In Fig. 5.1 we show the same route shown previously in Fig. 2.1 but now in the physical model. The interfering signals considered in the physical model, but not our model, are shown as thick curved lines.

The complement set of interferers contributes a nonnegative amount of interference. For a given $\mathbf{R}$ and $\mathbf{P}$ it follows that $\rho(\mathbf{R}, \mathbf{P}) \geq \hat{\rho}(\mathbf{R}, \mathbf{P})$. Equality between $\rho(\mathbf{R}, \mathbf{P})$ and $\hat{\rho}(\mathbf{R}, \mathbf{P})$ occurs only for $n \in\{1,2,3\}$ and $\hat{\rho}(\mathbf{R}, \mathbf{P})=\hat{r}_{1}$, or trivially when $\rho(\mathbf{R}, \mathbf{P})=0$. From the definition of the optimal throughput in both models, it follows that

$$
\hat{\rho}\left(\mathbf{R}^{*}, \mathbf{P}^{*}\right) \leq \hat{\rho}\left(\hat{\mathbf{R}}^{*}, \hat{\mathbf{P}}^{*}\right) \leq \rho\left(\mathbf{R}^{*}, \mathbf{P}^{*}\right)
$$

Thus our solution provides an upper and lower bound for the optimal solution in the physical model. Define the gap between the upper bound and the lower bound as

$$
\Delta=\rho\left(\mathbf{R}^{*}, \mathbf{P}^{*}\right)-\hat{\rho}\left(\mathbf{R}^{*}, \mathbf{P}^{*}\right)
$$

We now give a bound on the gap $\Delta$ that relates to a given instance of the network. Afterwards we analyze the asymptotic behavior of $\Delta$. For any $i \in\{1, \ldots, n\}$ and $\omega_{i} \leq 1$ define $\log \left(\omega_{i}\right)^{+}=0$, else if $\omega_{i}>1$ then $\log \left(\omega_{i}\right)^{+}=\log \left(\omega_{i}\right)$. It follows that any solution 
in our model for a network instance can be bounded above as

$$
\rho\left(\mathbf{R}^{*}, \mathbf{P}^{*}\right)<1+\log \left(\frac{P_{i} h_{i, i+1}}{1+I_{i+1}}\right)^{+} \forall i \in\{1, . ., n\},
$$

and for $k \in\{1, \ldots, n\}$ such that $\hat{\rho}\left(\mathbf{R}^{*}, \mathbf{P}^{*}\right)=r_{k}$ a lower bound for the solution from our algorithm in the physical model is

$$
\hat{\rho}\left(\mathbf{R}^{*}, \mathbf{P}^{*}\right)>\log \left(\frac{P_{k} h_{k, k+1}}{1+I_{k+1}+\sum_{\left\{j \in \mathbf{I}_{k}+1\right\}} P_{j} h_{j, k+1}}\right)^{+} .
$$

From (5.4) and (5.5) it follows that for a given network topology the value of (5.3) can be bounded above as

$$
\Delta<1+\log \left(1+\frac{\sum_{\left\{j \in \mathbf{I}_{k+1}\right\}} P_{j} h_{j, k+1}}{1+I_{k+1}}\right)
$$

where $k$ is defined as for (5.5). Although $\Delta$ can be calculated exactly when a network topology is given, (5.6) shows that $\Delta$ grows at most with the ratio between the interference from the complement set of interferers at $k$ and the interference considered by our model.

The complement set of interferers has maximum cardinality when considering reception at the destination. Therefore there are at most $n-1$ elements in the complement set of interferers and the maximum transmission power is $P_{\text {Max }}$. Then the upper bound of $\Delta$ can be extended to

$$
\Delta<1+\log \left(1+(n-1) P_{M a x}\right)
$$

and asymptotically grows as

$$
\Delta=O\left(\log (n-1)+\log \left(P_{\text {Max }}\right)\right) .
$$


Recall that $n$ is the number of transmitting nodes in a route and $N$ is the total number of nodes in the network. Therefore $n$ is not only bounded above by $N$, but the value of $n$ depends on the topology of all $N$ nodes.

\subsection{Performance of $\Delta$ with increasing $P_{M a x}$}

Now we analyze the behavior of $\Delta$ for a growing $P_{M a x}$. The analysis in this section is independent of network topology. We show in Lemma 5 that $n$ is inversely related to $P_{M a x}$, a result that arises from both self-interference and one hop interference. The inverse relation between $n$ and $P_{\text {Max }}$ is used to show that $\Delta$ tends to 0 for a sufficiently large $P_{\text {Max }}$.

Lemma 5: Consider any fixed network with $N$ nodes and an increasing $P_{\text {Max }}$. There exists some $P_{0}$ such that for every $P_{\text {Max }}>P_{0}$ the optimal route $\mathbf{R}^{*}$ has cardinality 2 meaning a direct transmission.

Proof. Consider an arbitrary $\mathbf{R}$, that is not a direct transmission, of cardinality $n^{\prime}+1$ and with an optimal power allocation. From Lemma 2 there exists a $j \in\left\{1, \ldots, n^{\prime}\right\}$ such that $P_{j}=P_{\text {Max }}$ for which the achievable rate will be

$$
r_{j}=\log \left(1+\frac{P_{M a x} h_{j, j+1}}{1+I_{j+1}}\right) .
$$

The maximum achievable rate for a direct transmission, and therefore the maximum throughput, is

$$
r_{S}=\log \left(1+P_{M a x} h_{S, D}\right)
$$

where the channel gain between source and destination, $h_{S, D}$, is fixed since $N$ is fixed. Therefore, the direct transmission rate grows with $P_{\text {Max }}$. Now consider the possible values of $j$, the node operating at $P_{\text {Max }}$, as $P_{\text {Max }}$ grows. If $j \neq 1$ then there exists 
an element $j-1$ that, from Lemma 1, can increase its transmission power without lowering throughput.

Assume that $j-1$ can increase its transmission without decreasing the throughput of the route. Any increase to the transmission power of $j-1$ that does not lower throughput is upper pounded by $P_{j-1}=P_{M a x}$. Consider $P_{j-1}=P_{M a x}$, then the equation

$$
r_{j-1}=\log \left(1+\frac{P_{M a x} h_{j-1, j}}{1+P_{M a x} \gamma_{j}+P_{j+1} h_{j, j+1}}\right),
$$

tends to a constant as $P_{\text {Max }}$ grows. Therefore any value of $P_{j-1}<P_{M a x}$ that does not lower throughput must also tend at most to the same constant.

Consider the case when from construction of $\mathbf{P}^{*}$ for $j=1$ the allocated power is such that $P_{j}=P_{M a x}$. Therefore we wish to show that

$$
r_{j}=\log \left(1+\frac{P_{M a x} h_{j, j+1}}{1+I_{j+1}}\right)<\log \left(1+P_{M a x} h_{S, D}\right),
$$

is true. We can rewrite both sides as

$$
\omega_{j+1}=\frac{P_{M a x} h_{j, j+1}}{1+I_{j+1}}<P_{M a x} h_{S, D}
$$

or as

$$
\frac{h_{j, j+1}}{h_{S, D}\left(1+I_{j+1}\right)}<1 .
$$

Due to rate equalization as $P_{M a x}$ grows, so should $P_{j+1}$ as well as $P_{j+2}$. Therefore, there exists a threshold value for which (5.14) is true. The exact value of this threshold depends on the channel gains and residual self-interference coefficient of the elements in the route as well as $h_{S, D}$.

Any route $\mathbf{R}$ operates at maximum throughput only with an optimal power allocation $\mathbf{P}^{*}$. In any $\mathbf{P}^{*}$ there exists one element $j \in\{1, \ldots, n\}$ such that $P_{j}=P_{\text {Max }}$ the 
arguments stated above hold for any possible route in the network.

Since (5.10) grows unbounded and any alternative route tends to at most a constant, it follows that $P_{0}$ exists. Therefore, at sufficiently large $P_{M a x}$ a direct transmission achieves a throughput higher than any other alternative route and thus $\mathbf{R}^{*}=\{S, D\}$.

Lemma 5 only proves the existence of $P_{0}$ since the value of $P_{0}$ depends on residual self-interference coefficients and channel gains in the network, in particular the channel gain $h_{S, D}$. The result from Lemma 5 can be used to infer that routing through several nodes may be optimal when $P_{M a x}$ is relatively low or moderate and a direct transmission is optimal when $P_{M a x}$ is sufficiently large. Theorem 3 uses Lemma 5 to show that at large $P_{\text {Max }}$ the value of $\Delta$ becomes zero.

Theorem 3: Consider a network with a fixed number of nodes $N$ and increasing $P_{\text {Max }}$. The value of $\Delta$ becomes zero for a sufficiently large value of $P_{\text {Max }}$.

Proof. From Lemma 5 it follows that a direct transmission is the optimal $\mathbf{R}^{*}$ for a sufficiently large $P_{\text {Max }}$. Sufficiently large $P_{\text {Max }}$ is such that $P_{\text {Max }}>P_{0}$, where $P_{0}$ is defined in Lemma 5. Therefore, at sufficiently large $P_{\text {Max }}$ the number of transmitters is $n=1$ for which the complement set of interferers is empty. For a direct transmission the complement set of interferers is empty, therefore $\rho\left(\mathbf{R}^{*}, \mathbf{P}^{*}\right)=\hat{\rho}\left(\mathbf{R}^{*}, \mathbf{P}^{*}\right)$ and $\Delta=0$.

From Theorem 3 it follows that as $P_{\text {Max }}$ grows then the optimal solution in our model tends to the optimal solution in the physical model. The result from Theorem 3 does not contradict the upper bound described in (5.6), since even though the transmit power may increase the number of interferers decreases. More so, Theorem 3 shows that $\Delta$ tends to zero as $P_{\text {Max }}$ grows, therefore so should the order of $(5.8)$. 


\subsection{Performance of $\Delta$ with increasing $N$ in a Linear Network}

Now we consider the case of a fixed $P_{\text {Max }}$ and growing $N$. To analyze the case of growing $N$ we consider the network to be a linear network. The use of linear networks offers a direct relation between $N$ and channel gains as well as limiting node placement to one dimension. Thus, a linear network offers structure into the topology which enables our analysis.

In a linear network all nodes are in the direct line joining the source and the destination with equal channel gains between each two consecutive nodes, as in $[11$, 23-25]. We assume that as $N$ grows the nodes $S$ and $D$ remain fixed and all other nodes are rearranged between them to maintain channel gain equality. We assume all nodes operate with the same residual self-interference coefficient $\gamma$.

Furthermore, we assume a path-loss model such that for $i, j \in\{1, \ldots, N\}$ channel gains are defined as $h_{i, j}=d_{i, j}^{-\alpha}$, where $d_{i, j}$ is the distance between node $i$ and node $j$ and the path-loss coefficient is $\alpha$. Therefore, in a linear network we define $h_{i, j}=$ $\left(d_{S, D} /(N-1)\right)^{-\alpha}$. Since for a value of $N$ all channel gains are equal, and growing with $N$, we use $h$ as the notation of a channel gain for linear network of size $N$.

Furthermore, assume that $n=N-1$, that is to say that all nodes in the linear network are selected into $\mathbf{R}$. For any $i, j \in\{1, \ldots, n\}$ with $i<j$ the order of $\mathbf{R}$ is such that there are fewer nodes in between $S$ and $i$ than the nodes in between $S$ and $j$. An example of a linear network is shown in Fig. 2.1.

Lemma 6 uses the recursive procedure used to construct $\mathbf{P}^{*}$ to show that the optimal power allocation for a linear network is such that a node in the route will operate at a lower transmission power than any node previous to it in the route. Lemma 7 then builds on both Lemma 2 and Lemma 6 to upper bound the throughput 
in a linear network that grows with $N$. We remind the reader that for a linear network $n=N-1$, but we use $n$ to relate to the transmitting nodes in a route and $N$ to relate to the total nodes in the network.

Lemma 6: Consider a linear network of size $N$ and the optimal power allocation $\mathbf{P}^{*}$. For all $i, j \in\{1, \ldots, n\}$ if $i<j$ then $P_{i}>P_{j}$.

Proof. Recall that for an optimal power allocation $r_{i}=r_{j} \forall i, j \in\{1, \ldots, n\}$, therefore if $i=n-1$ and $j=n$

$$
\log \left(1+\frac{P_{n-1} h}{1+P_{n} \gamma}\right)=\log \left(1+P_{n} h\right)
$$

Recall that in a linear network all channel gains between consecutive nodes are equal. Since $P_{n} \gamma$ is positive for imperfect self-interference cancellation, then $P_{n-1}>P_{n}$ to maintain equality among achievable rates. For any $i<n-1$ it follows that $I_{i}>I_{i+1}$. Therefore, generalizing for any $i<j \in\{1, \ldots, n\}$

$$
\log \left(1+\frac{P_{i} h}{1+I_{i+1}}\right)=\log \left(1+\frac{P_{j} h}{1+I_{j+1}}\right)
$$

since $I_{k} \forall k \in 1, \ldots, n$ is positive and $I_{i+1}>I_{j+1}$. Therefore to maintain rate equality, a property of $\mathbf{P}^{*}$, it follows that $P_{i}>P_{i+1}>\ldots>P_{j-1}>P_{j}$.

Combining the results from Lemma 2 and Lemma 6 it follows that for an optimal power allocation $P_{1}=P_{\text {Max }}$. The result in Lemma 6 comes from both the selfinterference and the one hop interference considered in the model.

In Lemma 7, a linear network with growing $N$ and fixed $P_{\text {Max }}$ is considered and the behavior of $\omega^{*}$ is explored. The exact value of $\omega^{*}$ depends on the value of $N$, the behavior of $h$ with $N, \gamma$, and $P_{M a x}$. Lemma 7 shows that there exists a function of $N$ that bounds $\omega^{*}$ from above. Lemma 7 also shows that at sufficiently large $N$ the 
function that bounds $\omega^{*}$ from above tends to a constant.

Lemma 7: Consider a linear network with fixed $P_{\text {Max }}$ and a growing number of nodes $N$. If the growth with $N$ of the channel gain between consecutive nodes is slower than an exponential growth, then the optimal throughput is bounded above by a function that decreases to a constant.

Proof. For any $i \in\{1, \ldots, n\}$ and $\omega^{*}$, the optimal SINR, (3.14) is used in our model to find the optimal transmission power $P_{i}$. We evaluate several terms to show a term that grows with $n$ due to recursivity. When $i=n$ then $P_{n}=\omega^{*} / h$. For $i=n-1$

$$
P_{n-1}=\frac{\omega^{*}}{h}+\gamma \frac{\omega^{* 2}}{h^{2}}
$$

the same $\omega^{*} / h$ term is present as in $P_{n}$. When $i=n-2$ then

$$
P_{n-2}=\frac{\omega^{*}}{h}+\gamma \frac{\omega^{* 2}}{h^{2}}+\gamma^{2} \frac{\omega^{* 3}}{h^{3}}+\frac{\omega^{* 2}}{h}
$$

In $(5.18)$ the term $\omega^{* 2} / h$ comes from the one hop interference originated at $n$. For $i=n-3$ due to one hop interference originating at $n-1$ the same $\omega^{* 2} / h$ term is present. For $i=n-4$ a term $\omega^{* 3} / h$ is present, again due to one hop interference now originating at $n-2$. From the previous example a recursive trend is visible.

The recursive term of interest is now generalized for any other element in the route. For any $j \in\{2, \ldots, n\}$ that is even then the optimal power allocation $P_{n-j}$ includes a term $\omega^{*(j / 2)} / h$. For any $k \in\{1, \ldots, n\}$ that is odd then the optimal power allocation $P_{n-k}$ includes a term $\omega^{*((k+1) / 2)} / h$. Without loss of generality assume $n$ is even. Therefore $P_{1}$ includes a term $\omega^{*(n / 2)} / h$. Recall that according to Lemma 2 and Lemma 6 , we have $P_{1}=P_{\text {Max }}$. Since all the terms in $P_{1}$ are positive it follows that

$$
P_{M a x}>\frac{\omega^{*(n / 2)}}{h}
$$


which can also be written as

$$
\left(P_{M a x} h\right)^{2 / n}>\omega^{*}
$$

Recall that $P_{M a x}$ is fixed therefore since $h$ grows with $N$ there exists some value of $N$ such that $P_{\text {Max }} h>1$. When $P_{\text {Max }} h>1$ then the left hand side of (5.20) decreases to one unless $h$ grows faster than exponentially with $N$. Therefore $\omega^{*}$ at sufficiently large $N$ is bounded above by one. Note that we can arrive to the same conclusion had we assumed $n$ was odd. Since $\omega^{*}$ is bounded above it follows that as $N$ grows, the throughput is also bounded above by a function that tends to a constant.

From Lemma 7 it follows that for $P_{M a x} \geq 1$ the upper bound on the throughput begins to decrease after channel gains are such that they amplify (i.e. $h>1$ ), rather than attenuate (i.e. $h<1$ ), the one hop interference. As $N$ grows and channel gains correspond to amplification channel gains then the upper bound on the throughput decreases to a constant. In Theorem 4 the result of Lemma 7 is used directly to prove another asymptotic behavior of $\Delta$.

Theorem 4: Consider a linear network with a fixed maximum transmission power $P_{\text {Max }}$. As $N$ grows then $\Delta$ tends at most to a constant.

Proof. From Lemma 7 it follows that at sufficiently large $N$ the throughput at most can increase towards the same constant to which the left hand side in (5.20) decreases. Therefore the value of $\rho\left(\mathbf{R}^{*}, \mathbf{P}^{*}\right)$ can at most tend towards that constant. Since the complement set of interferers increases with $N$ and the throughput in our model tends to a constant it follows that $\hat{\rho}\left(\mathbf{R}^{*}, \mathbf{P}^{*}\right)$ tend asymptotically to zero. Therefore $\Delta$ grows asymptotically towards the same constant as the left hand side in (5.20).

It is important to note that once channel gains correspond to amplification channels the path-loss model may not be a suitable model. To avoid entering the regime 
corresponding to amplification channels we can bound $h \leq 1$. Doing so increases the value tended to by function that upper bounds the throughput, thus increasing the value of $\Delta$. While $\Delta$ may increase it would still remain constant for sufficiently large $N$.

While Theorem 3 and Theorem 4 give insight into the validity of our model for asymptotically growing network characteristics, $N$ and $P_{M a x}$, the performance analysis of our model in moderate scenarios is left to simulations in the following section. 
Chapter 6

\section{Numerical Results}

Through simulations we evaluate the performance of the optimal route and power allocation found by our algorithm in a wireless network with imperfect full-duplex nodes as described in Chapter 2. We simulate a path-loss dominated network such that channel gains are defined as $h_{i, j}=d_{i, j}^{-\alpha}$, where $d_{i, j}$ is the distance between node $i$ and node $j$ and the path-loss coefficient is $\alpha$. Nodes have equal capabilities, therefore the residual self-interference coefficients are set to $\gamma_{i}=\gamma \forall i$. We select the value of $\gamma=0.01$, which corresponds to $20 \mathrm{~dB}$ gap between received signal and residual selfinterference, as reported in [12], while $\gamma=0.03$ corresponds to approximately a $15 \mathrm{~dB}$ gap and $\gamma=0.20$ to approximately a $7 \mathrm{~dB}$ gap. Results for optimal route and power allocation half-duplex and for direct transmission are also shown for comparison. Half-duplex is when a node is not able to simultaneously transmit and receive over the same frequency. Results shown are averaged from 500 simulation results.

In Fig. 6.1, Fig. 6.2, Fig. 6.3, and Fig. 6.4 the number of nodes in the network is set to $N=20$. Nodes $S$ and $D$ have fixed positions at 10 meters apart from each other and the rest are randomly placed in a square area of 20 meters side length. Node placement is done this way such that direct transmission becomes comparable to full-duplex at high $P_{M a x}$. 


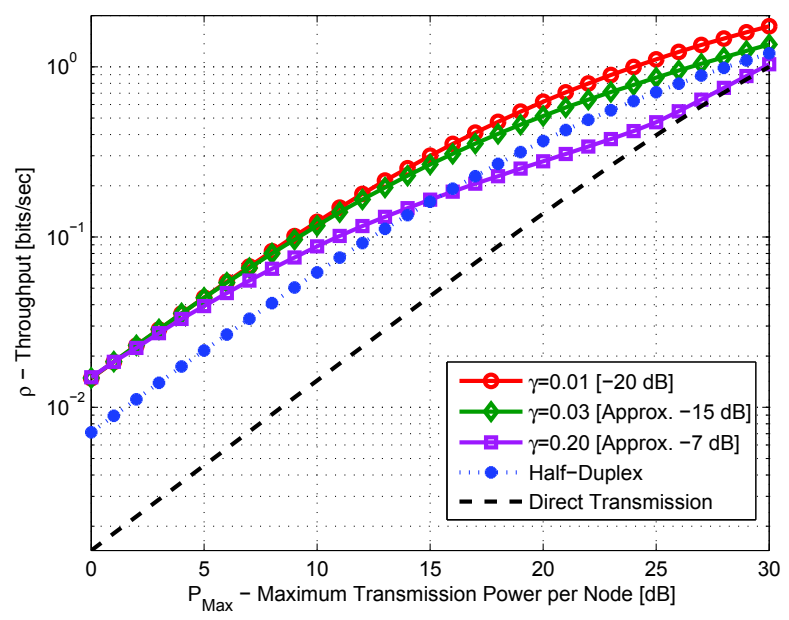

Figure 6.1: Achieved throughput for optimal route and power allocation with $\alpha=3$. Throughput is represented on a logarithmic scale.

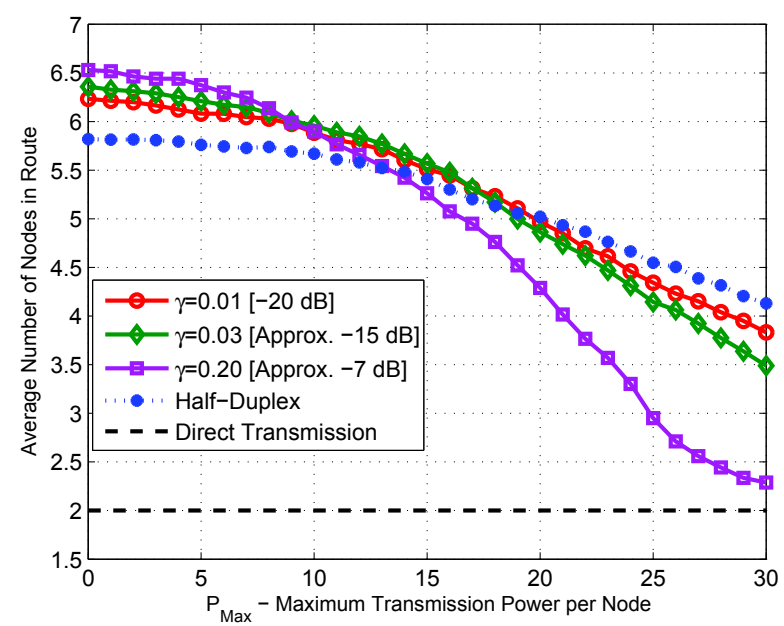

Figure 6.2: Average number of nodes in the optimal route as a function of $P_{\text {Max }}$ for a fixed network size and for path-loss coefficient $\alpha=3$.

Using our model the average throughput, on a logarithmic scale, for the optimal route and power allocation as a function of $P_{\text {Max }}$ with $\alpha=3$ is shown in Fig. 6.1. In our model as long as interference cancellation is imperfect, half-duplex outperforms full-duplex for a sufficiently high $P_{\max }$, such as the case of $\gamma=0.03$ being outperformed past $P_{\text {Max }}>15 \mathrm{~dB}$. As $P_{\text {Max }}$ grows so does the residual self-interference and the one hop interference because, as Lemma 2 states, there is at least one node is 


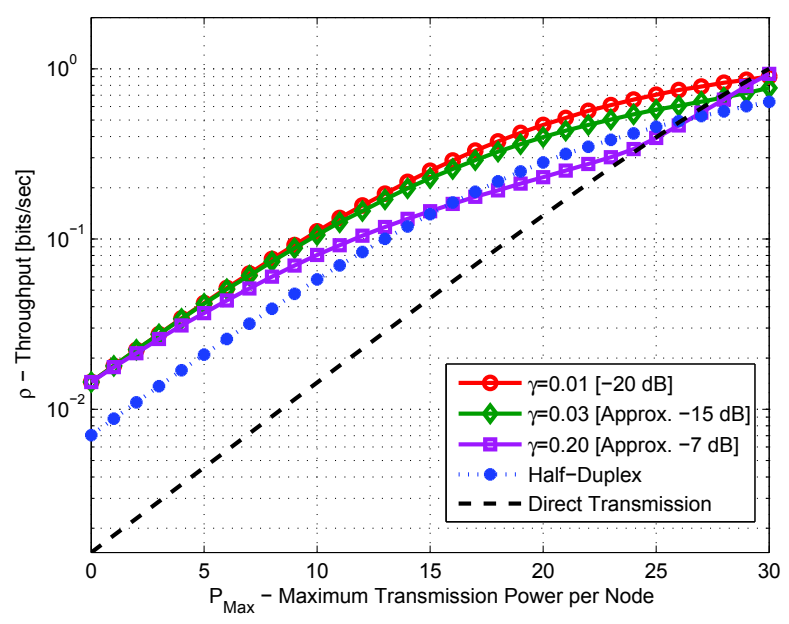

Figure 6.3: Solution from Fig. 6.1 in model including interferences from all transmitting nodes. Throughput is represented on a logarithmic scale.

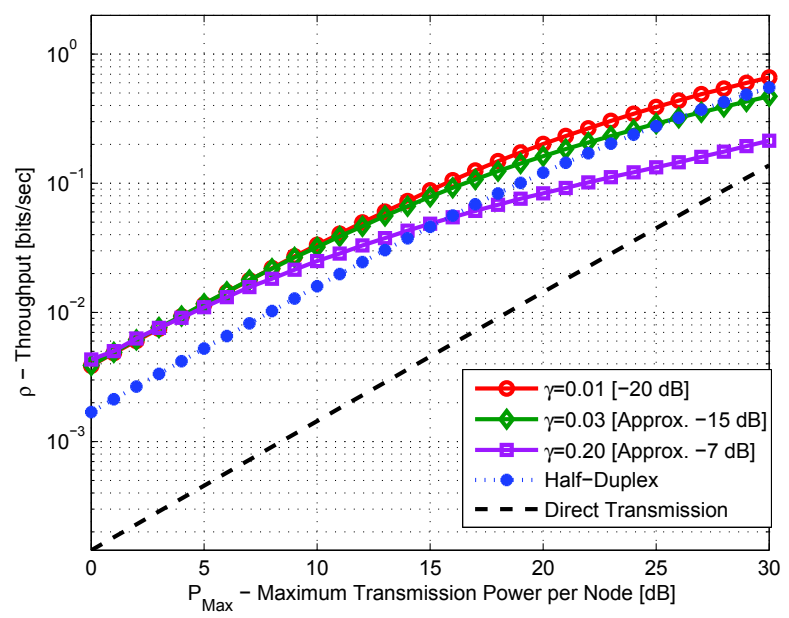

Figure 6.4: Achieved throughput for optimal route and power allocation with $\alpha=4$. Throughput is represented on a logarithmic scale.

transmitting at $P_{\text {Max }}$.

To see the effects of increased interference on route selection in our model refer to Fig. 6.2 where the average number of nodes in the optimal route as a function of $P_{M a x}$ are shown. In particular, for high $P_{\text {Max }}$ the average number of nodes in the optimal route for $\gamma=0.03$ tends to 2 . This route selection is expected at higher $P_{\text {Max }}$ for other values of $\gamma$. Therefore, from Fig. 6.1 and Fig. 6.2, it is clear that for large 
amounts of interference are present the optimal route reduces to direct transmission. The results shown in Fig. 6.2 also follow the result found for increasing $P_{M a x}$ and fixed $N$ in the previous section.

Recall that our model simplifies interference to be only self-interference and onehop interference. To examine the degree to which this simplification affects performance, we relax this assumption. After solving for the optimum routes and power allocations for our simplified model, we compute the resulting throughput in the physical model which is a more complete model. In the physical model assumes that each node interferes with every other node according to the path-loss model described above. This corresponds to the lower bound on the optimal throughput for the physical model, as described in Chapter 5. We plot the computed throughput in Fig. 6.3. For $\gamma=0.01$ throughput falls sharply for $P_{M a x} \geq 20 \mathrm{~dB}$, since the routes selected tend to be longer for which many small interference components combine, resulting in a large decrease in throughput. For higher $\gamma$, however, the difference between Fig. 6.1 and Fig. 6.3 is smaller, since the optimal routes alleviate interference more conservatively by choosing shorter routes. On average the solutions in Fig. 6.1 decrease for $\gamma=0.01$ by $19 \%$, for $\gamma=0.03$ by $18 \%$ and for $\gamma=0.20$ by $11 \%$ when in the more complete model.

To see the effects of $\alpha$ in Fig. 6.4 the average throughput, on a logarithmic scale, for the optimal route and power allocation as a function of $P_{\text {Max }}$ with $\alpha=4$ is shown. The computation of our solution in a more complete model is not shown since the plots are very similar. The solutions in Fig. 6.4 when computed for all interferers decrease on average, across the entire range shown, for $\gamma=0.01$ by $9 \%$, for $\gamma=0.03$ by $9 \%$, and for $\gamma=0.20$ by $7 \%$. Therefore, it can be inferred that our model offers a performance closer to the optimal solution in the physical model when larger values of the path-loss coefficient are considered. The discrepancy in throughput between our 


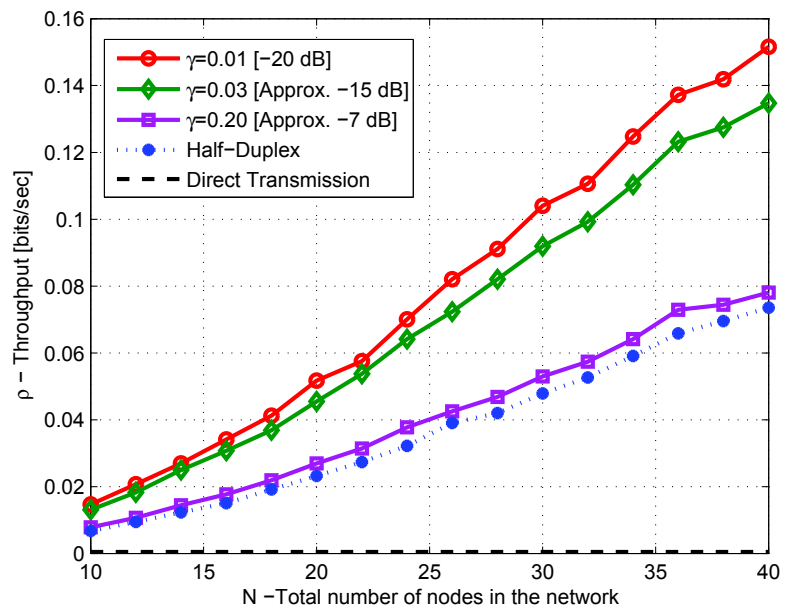

Figure 6.5: Achieved throughput for optimal route and power allocation with $\alpha=4$.

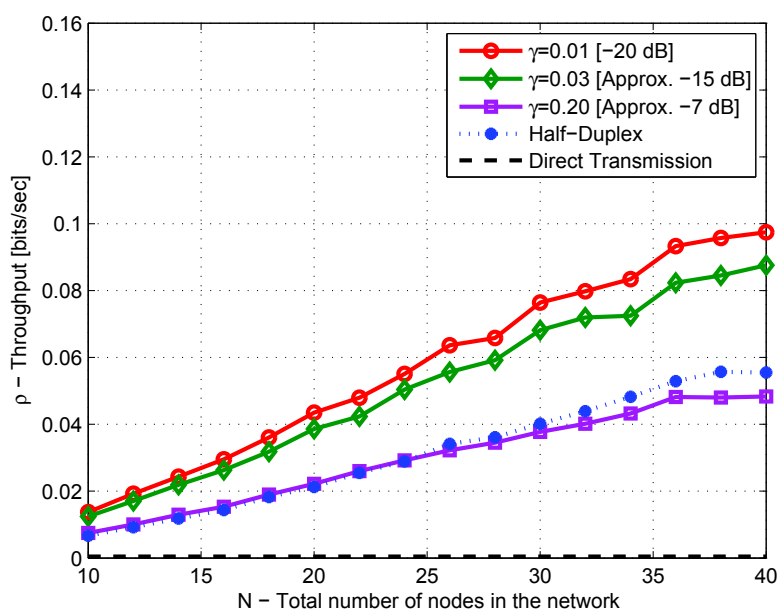

Figure 6.6: Performance of our solution in a model including interferences from all transmitting nodes with $\alpha=4$.

model and the physical model increases for lower values of the path-loss coefficient, which represents ideal and unrealistic channels.

We also evaluate achieved throughput while varying $N$ and fixing $P_{\text {Max }}=15 \mathrm{~dB}$. For these simulations $S$ and $D$ are set 18 meters apart. This was done such that longer routes become more common. Results obtained in our model are shown in Fig. 6.5 and the throughput recalculated for a more complete model is shown in Fig. 6.6. 
In Fig. 6.5 half-duplex is outperformed, since $P_{\text {Max }}$ is not sufficiently high, and the throughput grows with $N$. The throughput discrepancy between our simplified model and the more complete model increases with $N$. In Fig. 6.6 at higher values of $N$ half-duplex outperforms $\gamma=0.20$ because a larger network tends to a longer optimal route; hence there is a greater amount of neglected interference, resulting in a greater impact to throughput. As shown in Fig. 6.5 and Fig. 6.6, however, the discrepancy is small for moderate $N$. Our approach therefore represents a tradeoff, reducing the complexity of finding a solution considerably while providing slightly sub-optimal performance in the more complete model. 
Chapter 7

\section{Conclusion}

An algorithm is proposed that finds both the routing and power allocation problem in an imperfect full-duplex network to maximize the throughput of a set source and destination. In particular we present a model that considers imperfect self-interference cancellation, hence the imperfect full-duplex operation, and one hop interference. Based on this model we solve the problem for when the route is given, finding a necessary condition for optimality and a procedure to find the optimal power allocation. The procedure has a complexity similar to a polynomial root finding algorithm, where the highest degree of the polynomial is equal to the number of transmitting nodes in a route. We propose a priority-first search algorithm that finds both the route and the power allocation for which the throughput is maximized.

Our algorithm differs from Dijkstra's algorithm by having a metric that is coupled to the decisions and non decomposable, a property that arises from the interference model. The performance of the solution found in our model is shown by analyzing the gap between a solution in our model and the optimal solution in the physical model. The gap between solutions is bounded and shown to tend asymptotically to zero as more transmission power is available. In a linear network the gap is shown to tend asymptotically to a constant. 
While $\Delta$ is shown to decrease to, at most, a constant the performance for moderate values of maximum transmit power and number of wireless nodes is inspected through simulation. Through simulations it is shown that even under imperfect selfinterference cancellation multi-hop routing in full-duplex outperforms, in terms of throughput, operating in half-duplex. The gap between the throughput in our model and a more complete model is found to increase with $N$ and decrease with an increase in $\alpha$. This gap comes from using our model, the same model that allows finding a solution with complexity similar to finding polynomial roots. When considering our algorithm it is important to note that our solution requires less network information than the network information requried to find the optimal solution in the physical model. 


\section{Bibliography}

[1] X. Lin and N. Shroff, "Joint rate control and scheduling in multihop wireless networks," in Decision and Control, 2004. CDC. 43rd IEEE Conference on, pp. 1484 - 1489 Vol.2, Dec. 2004. 1, 2.3

[2] R. Cruz and A. Santhanam, "Optimal routing, link scheduling and power control in multihop wireless networks," in INFOCOM 2003. Twenty-Second Annual Joint Conference of the IEEE Computer and Communications. IEEE Societies, March 2003. 1, 2.3

[3] M. Chiang, "Balancing transport and physical layers in wireless multihop networks: jointly optimal congestion control and power control," Selected Areas in Communications, IEEE Journal on, Jan. 2005. 1, 2.3

[4] S. Kompella, J. Wieselthier, A. Ephremides, and H. Sherali, "A cross-layer approach to end-to-end routing and sinr-based scheduling in multi-hop wireless networks," in WiOPT 2008, pp. 261 -266, April 2008. 1, 2.3

[5] L. Ong, M. Motani, and S. J. Johnson, "On capacity and optimal scheduling for the half-duplex multiple-relay channel," Information Theory, IEEE Transactions 
on, vol. 58, Sept. 2012. 1, 2.3

[6] N. Badruddin, S. Hanly, and J. Evans, "Optimal binary power allocation for wireless networks with local interference," in Communications (ICC), 2010 IEEE International Conference on, pp. 1 -5, May 2010. 1, 2.3

[7] P. Thulasiraman, J. Chen, and X. Shen, "Max-min fair multipath routing with physical interference constraints for multihop wireless networks," in Communications, 2010 IEEE International Conference on, May 2010. 1, 2.3

[8] T. ElBatt and A. Ephremides, "Joint scheduling and power control for wireless ad hoc networks," Wireless Communications, IEEE Transactions on, vol. 3, pp. 74 - 85, jan. 2004. 1, 2.3

[9] S. Weber, J. Andrews, X. Yang, and G. de Veciana, "Transmission capacity of wireless ad hoc networks with successive interference cancellation," Information Theory, IEEE Transactions on, vol. 53, pp. 2799 -2814, Aug. 2007. 1, 2.3

[10] Z. Jia, R. Gupta, J. Walrand, and P. Varaiya, "Bandwidth guaranteed routing for ad-hoc networks with interference consideration," in Computers and Communications, 2005. ISCC 2005. Proceedings. 10th IEEE Symposium on, pp. 3 9 , june 2005. 1, 2.3

[11] L. Dritsoula and C. Papadias, "On the throughput of linear wireless multi-hop networks using directional antennas," in Signal Processing Advances in Wireless Communications, 2009. SPAWC '09. IEEE 10th Workshop on, pp. $384-388$, june 2009. 1, 2.3, 5.3

[12] M. Duarte and A. Sabharwal, "Full-duplex wireless communications using offthe-shelf radios: Feasibility and first results," in Signals, Systems and Computers 
(ASILOMAR), 2010 Conference Record of the Forty Fourth Asilomar Conference on, pp. $1558-1562$, Nov. 2010. 1, 2.1, 2.1, 2.3, 6

[13] M. Jain, J. I. Choi, T. Kim, D. Bharadia, S. Seth, K. Srinivasan, P. Levis, S. Katti, and P. Sinha, "Practical, real-time, full duplex wireless," in Proceedings of the 17th annual international conference on Mobile computing and networking, MobiCom '11, ACM, 2011. 1, 2.3

[14] J. I. Choi, M. Jain, K. Srinivasan, P. Levis, and S. Katti, "Achieving single channel, full duplex wireless communication," in MOBICOM'10, 2010. 1, 2.3

[15] B. Radunovic, D. Gunawardena, P. Key, A. Proutiere, N. Singh, V. Balan, and G. Dejean, "Rethinking indoor wireless mesh design: Low power, low frequency, full-duplex," in Wireless Mesh Networks (WIMESH 2010), 2010 Fifth IEEE Workshop on, pp. 1 -6, June 2010. 1, 2.3

[16] T. Riihonen, S. Werner, R. Wichman, and Z. Eduardo, "On the feasibility of full-duplex relaying in the presence of loop interference," in Signal Processing Advances in Wireless Communications, 2009. SPAWC '09. IEEE 10th Workshop on, pp. $275-279$, June 2009. 2.3

[17] T. Riihonen, S. Werner, and R. Wichman, "Hybrid full-duplex/half-duplex relaying with transmit power adaptation," Wireless Communications, IEEE Transactions on, vol. 10, September 2011. 2.3

[18] K. Yamamoto, K. Haneda, H. Murata, and S. Yoshida, "Optimal transmission scheduling for a hybrid of full- and half-duplex relaying," Communications Letters, IEEE, vol. 15, March 2011. 2.3 
[19] X. Fang, D. Yang, and G. Xue, "Distributed algorithms for multipath routing in full-duplex wireless networks," in Mobile Adhoc and Sensor Systems (MASS), 2011 IEEE 8th International Conference on, Oct. 2011. 2.3

[20] N. Singh, D. Gunawardena, A. Proutiere, B. Radunovic, H. Balan, and P. Key, "Efficient and fair mac for wireless networks with self-interference cancellation," in Modeling and Optimization in Mobile, Ad Hoc and Wireless Networks (WiOpt), pp. $94-101$, May 2011. 2.3

[21] C. Weeraddana, M. Codreanu, M. Latva-aho, and A. Ephremides, "Resource allocation for cross-layer utility maximization in multi-hop wireless networks in the presence of self interference," in Modeling and Optimization in Mobile, Ad Hoc and Wireless Networks (WiOpt), 2010 Proceedings of the 8th International Symposium on, June 2010. 2.3

[22] P. Gupta and P. Kumar, "The capacity of wireless networks," Information Theory, IEEE Transactions on, vol. 46, pp. 388 -404, Mar 2000. 1, 2.3, 5.1

[23] Y. Xiao and L. J. Cimini, "Maximal throughput routing in wireless linear networks," in Information Sciences and Systems (CISS), 2012 46th Annual Conference on, pp. 1 -6, march 2012. 2.3, 5.3

[24] D. Chiarotto, O. Simeone, and M. Zorzi, "Throughput and energy efficiency of opportunistic routing with type-i harq in linear multihop networks," in Global Telecommunications Conference (GLOBECOM 2010), 2010 IEEE, pp. 1 -6, dec. 2010. $2.3,5.3$

[25] P. MomČilović and M. S. Squillante, "On throughput in linear wireless networks," in Proceedings of the 9th ACM international symposium on Mobile ad 
hoc networking and computing, MobiHoc '08, (New York, NY, USA), pp. 199208, ACM, 2008. 2.3, 5.3

[26] S. M. Harb and J. Mcnair, "Analytical study of the expected number of hops in wireless ad hoc network," in Proceedings of the Third International Conference on Wireless Algorithms, Systems, and Applications, WASA '08, (Berlin, Heidelberg), pp. 63-71, Springer-Verlag, 2008. 3.2

[27] L. C. H. Feng, "On the optimum number of hops in a multi-hop linear network with randomly located nodes," in Proceeding of IEEE International Conference on Communications (ICC), June 2012. 3.2

[28] G. Sitton, C. Burrus, J. Fox, and S. Treitel, "Factoring very-high-degree polynomials," Signal Processing Magazine, IEEE, vol. 20, pp. 27 - 42, nov. 2003. 3.2

[29] V. Y. Pan, "Solving a polynomial equation: Some history and recent progress," SIAM Review, vol. 39, no. 2, pp. 187-220, 1997. 3.2

[30] E. W. Dijkstra, "A note on two problems in connexion with graphs," Numerische Mathematik, vol. 1, pp. 269-271, 1959. 4.1

[31] T. H. Cormen, C. Stein, R. L. Rivest, and C. E. Leiserson, Introduction to Algorithms. McGraw-Hill Higher Education, 2nd ed., 2001. 4.1

[32] D. Bertsekas and R. Gallager, Data Networks. Upper Saddle River, NJ, USA: Prentice-Hall, Inc., 1992. 4.1

[33] W. J. Cook, W. H. Cunningham, W. R. Pulleyblank, and A. Schrijver, Combinatorial optimization. New York, NY, USA: John Wiley \& Sons, Inc., 1998. 4.1 